\title{
Association of systemic comorbidity in diabetic serous macular detachment and comparison of various combination therapies in its management
}

This article was published in the following Dove Press journal:

Clinical Ophthalmology

II January 2013

Number of times this article has been viewed

\author{
Manoj Soman' \\ Sunil Ganekal ${ }^{2}$ \\ Unnikrishnan Nair' \\ KGR Nair' \\ 'Chaithanya Eye Hospital and \\ Research Institute, Trivandrum, \\ Kerala, ${ }^{2}$ Nayana Superspecialty Eye \\ Hospital and Research Center, \\ Davangere, Karnataka, India
}

Background: The purpose of this research was to study the association between systemic comorbidity in diabetic serous macular detachment (DSMD) and the effect of different forms of combination therapies in its management.

Methods: In this prospective analysis, 34 eyes from 34 patients with DSMD were investigated for the presence of systemic comorbidity including anemia, dyslipidemia, nephropathy, and cardiac disease, and treated with combination therapy of either intravitreal bevacizumab + laser (group $1, \mathrm{n}=14$ ) or intravitreal triamcinolone + laser (group 2, $\mathrm{n}=20$ ). Sequential macular laser was done 2 weeks after intravitreal pharmacotherapy in both groups. Outcome measures included visual acuity and central foveal thickness at 1 and 3 months.

Results: The mean age of the patients was $55.6 \pm 7.6$ years. The commonest systemic association was nephropathy $(82.3 \%)$. In group 1 , mean visual acuity improved marginally from $6 / 17$ at baseline to $6 / 16$ at 1 month $(P=0.0001)$ and was maintained at 3 months $(P=0.008)$; and mean central foveal thickness decreased from $488.7 \mu \mathrm{m}$ to $318.7 \mu \mathrm{m}$ at 1 month $(P=0.0001)$ but increased to $414.4 \mu \mathrm{m}$ at 3 months $(P=0.049)$. In group 2 , mean visual acuity improved from $6 / 22$ at baseline to $6 / 19$ at 1 month $(P=0.0001)$ and $6 / 12$ at 3 months $(P=0.0001)$; and mean central foveal thickness decreased from $428.8 \mu \mathrm{m}$ to $323.8 \mu \mathrm{m}$ at 1 month $(P=0.0001)$ to $269.2 \mu \mathrm{m}$ at 3 months $(P=0.0001)$.

Conclusion: Nephropathy should be ruled out in patients with DSMD. Although at 1 month both intravitreal triamcinolone and bevacizumab improved vision and decreased central foveal thickness in eyes with DSMD when administered along with focal laser treatment, the former had a more long-lasting effect in maintaining this gain at 3 months.

Keywords: diabetic serous macular detachment, nephropathy, combination therapy

\section{Introduction}

Macular edema is a frequent manifestation of diabetic retinopathy and an important cause of impaired vision in individuals with diabetes. It has been estimated that after 20 years of known diabetes, the prevalence of diabetic macular edema is approximately $28 \%$ in both type 1 and type 2 diabetes. ${ }^{1}$

The Early Treatment Diabetic Retinopathy Study (ETDRS) demonstrated that focal or grid laser photocoagulation for clinically significant diabetic macular edema reduced moderate visual loss by $50 \%$. However, $12 \%$ of treated eyes had still lost 15 or more ETDRS letters at 3-year follow-up and $24 \%$ of immediately treated eyes had thickening involving the center of the macula at 36 months. ${ }^{1}$ This suggests the existence of a distinct subgroup of eyes with diabetic macular edema resistant to conventional laser photocoagulation. With the advent of optical coherence tomography, it is now
Correspondence: Manoj Soman Chaithanya Eye Hospital and Research Institute, Trivandrum, Kerala 695004, India

$\mathrm{Tel}+9|47| 2447183$

Fax +9| 47I 2443985

Email soman.manoj@gmail.com 
possible to quantify and anatomically characterize significant diabetic macular edema. Optical coherence tomography patterns ${ }^{2,3}$ for clinically significant diabetic macular edema has been reported by various authors, and include sponge-like swelling, retinal edema with cystic spaces, retinal edema with submacular detachment (DSMD), and vitreomacular tractionassociated macular edema. Studies have shown that macular thickening due to retinal edema with cystic spaces, DSMD, or vitreomacular traction-associated macular edema carries a poor prognosis despite laser photocoagulation. , $^{4-6}$

Grid laser photocoagulation in a severely edematous macula is not only technically difficult but is also less effective in achieving the desired result. ${ }^{11}$ Recent studies have demonstrated the efficacy of various alternate therapy/combination therapies, especially in refractory clinically significant diabetic macular edema. Studies have also shown that, in addition to poor glycemic control, systemic comorbidity, such as anemia, dyslipidemia, nephropathy, or cardiac disease, has a major role in the pathogenesis of the disease, ${ }^{8,9}$ especially in eyes with diffuse macular edema. Submacular detachment is not a common feature on optical coherence tomography in eyes with significant diabetic macular edema. Ozdek et $\mathrm{al}^{6}$ found that $6.2 \%$ of eyes with submacular detachment during their analysis of 195 eyes with significant diabetic macular edema had a poor visual outcome after conventional treatment. A specific systemic association between this form of significant diabetic macular edema and management outcomes has not been studied, but is already well known with other forms of diabetic macular edema. We believed that this subset of eyes is different from the other tomographic forms of significant diabetic macular edema because they respond poorly to conventional laser therapy, unlike eyes with retinal edema with cystic spaces or spongy macular edema. Therefore, this study was undertaken to study eyes with DSMD.

\section{Materials and methods}

This was a prospective study of 34 eyes from 34 diabetic individuals with significant diabetic macular edema and submacular serous detachment. These 34 eyes (11.97\%) were from 284 eyes with significant diabetic macular edema who were already being studied. The study was approved by our institutional review board. Each eligible patient received full verbal and written information concerning the study drugs, and informed consent was taken. Significant diabetic macular edema was diagnosed on biomicroscopy as defined by ETDRS. Submacular detachment was defined on optical coherence tomography as the presence of fluid in the subretinal space under the fovea. Eyes with associated optical coherence tomography findings, such as retinal edema with cystic spaces and spongy edema, were also included. Because an intravitreal steroid (triamcinolone acetonide) was used in the management, eyes with glaucoma or ocular hypertension were excluded. Eyes with macular ischemia detected on fluorescein angiography or with vitreomacular traction on optical coherence tomography were excluded because these may be confounding variables with regard to the final outcome, especially for vision and central foveal thickness. Although eyes with vitreomacular traction were excluded, eyes with detached hyaloid at the macula without traction as evidenced by maintained foveal contour were included. Patients who had suffered a cardiovascular event or cerebrovascular accident in the previous 6 months were excluded. All patients underwent visual acuity estimation using the Snellen visual acuity chart, slit-lamp biomicroscopy, fundus fluorescein angiography, and optical coherence tomography. A detailed history and investigation for the presence of systemic comorbidity, including anemia, dyslipidemia, nephropathy, and cardiac disease, was done. Other associated causes of anemia including that after nephropathy were included. Systemic evaluation of the enrolled patients was performed by an inhouse physician. Patients were randomized to receive either intravitreal triamcinolone acetonide or intravitreal bevacizumab at baseline, followed by focal laser treatment after 2 weeks. Patients who received intravitreal triamcinolone acetonide were instructed to remain in the sitting position for at least 3 hours after the procedure and this facilitated early macular laser at 2 weeks following injection. Systemic and ocular risk factors associated with both forms of intravitreal injection were clearly explained to the patients. Standard protocols were followed for the injections.

The purpose of the study was not to compare the effect of intravitreal triamcinolone acetonide and bevacizumab, but to compare combination therapies. The purpose of intravitreal pharmacotherapy was to bring down the submacular detachment component of the edema in order to facilitate laser therapy. Because this study was designed to study the effect of combination therapy in eyes with submacular detachment, no further anti-vascular endothelial growth factor (VEGF) injections were given, even though it was known that their effect lasts only up to 8 weeks when given as monotherapy. However, there are a few reports of longer effects even with a single anti-VEGF injection when given along with laser therapy..$^{10}$ Patients were followed up after laser treatment at monthly intervals until 3 months. At each visit, visual acuity, 
intraocular pressure, findings on biomicroscopy, indirect ophthalmoscopy, and optical coherence tomography, and any adverse ocular or systemic events were documented. Statistical analysis was performed using the Statistical Package for Social Sciences version 19.0 (SPSS Inc, Chicago, IL). A $P$ value of 0.05 was considered to be statistically significant.

\section{Results}

The mean age of the patients was 55.6 \pm 7.6 (range 42-66) years. There were 22 males and 12 females, who had a mean duration of diabetes of 11.2 (range 3-16) years. The mean $\mathrm{HbA}_{1 \mathrm{c}}$ value was 7.9 (range 6.9-9). The study involved 14 eyes with moderate nonproliferative diabetic retinopathy (NPDR), 13 eyes with severe NPDR, and seven eyes with early proliferative diabetic retinopathy (PDR).

Twenty-eight of the 34 patients $(82.4 \%)$ had overt diabetic nephropathy or microalbuminuria. Of these, $11(39.3 \%)$ had known diabetic renal disease and were on treatment, including two patients on renal dialysis for end-stage disease. The rest $(60.7 \%)$ were found to have significant microalbuminuria/gross albuminuria after biochemical investigations done at presentation. Thus, this group was first identified to have renal disease on the basis of an ocular finding of subfoveal detachment. Twelve of the 34 patients $(35.3 \%)$ had anemia, $12(35.3 \%)$ had associated hypertension, and 11 (32.4\%) had dyslipidemia (Figure 1). Thus, diabetic nephropathy was the commonest association in diabetic subretinal detachment. Other features on optical coherence tomography included retinal edema with cystic spaces and spongy edema. Twenty-two eyes (64.7\%) had retinal edema with cystic spaces and $30(88.2 \%)$ had spongy edema in addition to foveal detachment.

Mean baseline visual acuity was 6/20 (range 6/12-6/36), which improved to $6 / 18$ after intravitreal bevacizumab/ triamcinolone and focal laser at 1 month and further to $6 / 14$ at 3 months. Subgroup analysis revealed that mean visual acuity in group 1 improved from $6 / 17$ at baseline to $6 / 15$ at 1 month

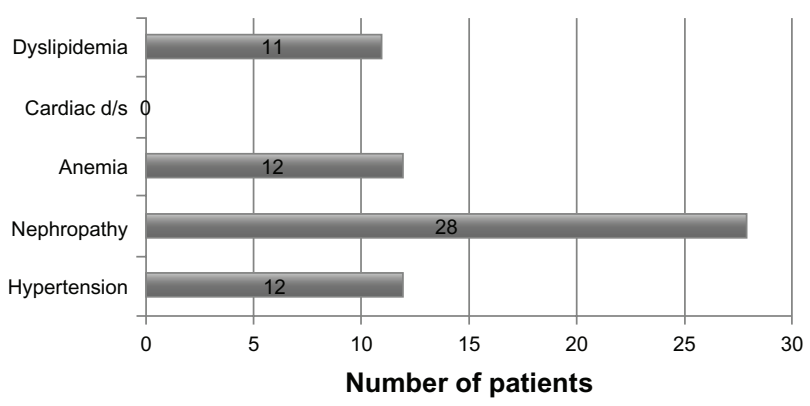

Figure I Various systemic comorbidities associated with diabetic serous macular detachment.
$(P=0.0001)$, but decreased to $6 / 16$ at 3 months $(P=0.080)$. Mean visual acuity in group 2 improved from $6 / 22$ at baseline to $6 / 19$ at 1 month $(P=0.0001)$, and further to $6 / 12$ at 3 months $(P=0.0001)$. Thus, although both intravitreal bevacizumab and triamcinolone improved vision in eyes with DSMD when administered with focal laser treatment at 1 month (shortterm efficacy), the former had a more long-lasting effect on maintaining this visual gain, as seen at 3 months (Figure 2).

Mean baseline central foveal thickness was 453.5 (range 346-590) $\mu \mathrm{m}$, which decreased significantly to $321.7 \mu \mathrm{m}$ after intravitreal bevacizumab/triamcinolone and focal laser at 1 month, but increased marginally to $329 \mu \mathrm{m}$ at 3 months. Subgroup analysis revealed that mean central foveal thickness in group 1 improved significantly from $488.7 \mu \mathrm{m}$ (confidence interval [CI] 511.34-466.057) at baseline to $318.7 \mu \mathrm{m}$ (CI 302.53-334.87) at 1 month $(P=0.0001)$ but increased to 414.4 $\mu \mathrm{m}$ (CI 389.9-438.8) at 3 months $(P=0.049)$. Mean central foveal thickness in group 2 improved significantly from $428.8 \mu \mathrm{m}$ (CI 390.08-467.52) at baseline to $323.8 \mu \mathrm{m}$ (CI 299.06-340.5) at 1 month $(P=0.0001)$ and further to 269.2 $\mu \mathrm{m}$ (CI 249.48-288.92) at 3 months $(P=0.0001)$. Thus, although both intravitreal triamcinolone and bevacizumab decreased macular thickening in eyes with DSMD when administered with focal laser at 1 month, the former had a more long-lasting effect on maintaining central foveal thickness, as seen at 3 months (Figure 3).

Submacular fluid absorption was complete in six of the 14 eyes $(42.9 \%)$ treated with intravitreal bevacizumab and nine of the 20 eyes $(45 \%)$ treated with intravitreal triamcinolone. These eyes had the best visual gain and also the best reduction in macular thickness. However, visual gain and reduction of macular thickness did not correlate statistically with reduction in the amount of submacular fluid. There were no ocular or systemic complications noted in either group.

\section{Discussion}

The mean duration of diabetes among our subjects was 11.2 years, and mean $\mathrm{HBA}_{1 \mathrm{c}}$ was 7.9 , so DSMD was

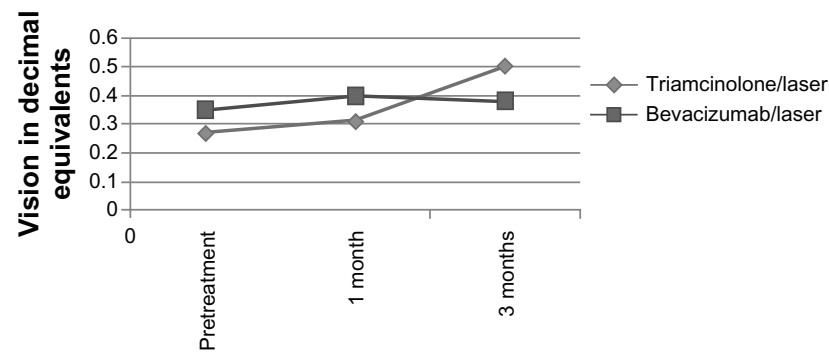

Figure 2 Mean change in visual acuity in both treatment groups over 3 months. 


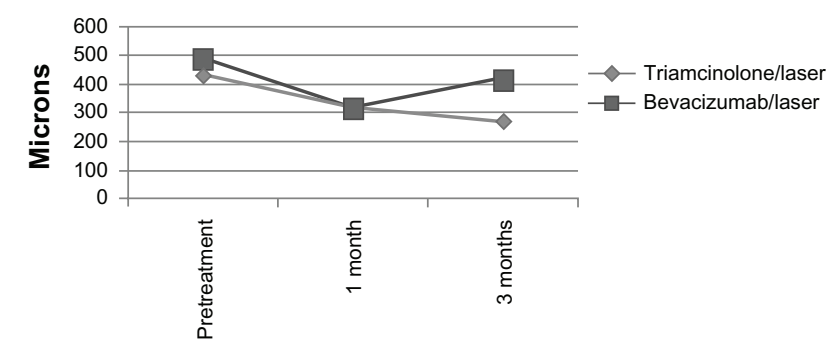

Figure 3 Mean change in central foveal thickness in both treatment groups over 3 months.

correlated with a longer duration of diabetes and poor glycemic control. Fourteen eyes with DSMD had moderate NPDR, 13 eyes had severe NPDR, and seven eyes had early PDR, indicating that DSMD did not correlate with the severity of background retinopathy. However, this observation could be due to selection bias, because eyes with macular ischemia were excluded from the study. Our unpublished data showed subfoveal detachment/DSMD in $14 \%$ of eyes with PDR and $3 \%$ of eyes with NPDR. Alkuraya et al ${ }^{9}$ investigated the correlation between features of macular edema on optical coherence tomography and the severity of retinopathy, and found that the prevalence of spongy edema was significantly higher in eyes with mild to moderate NPDR. DSMD and vitreomacular traction-associated macular edema were significantly more common in eyes with severe NPDR and PDR, and retinal edema with cystic spaces was seen in both groups. The fact that DSMD was seen in patients with long-standing diabetes and poor glycemic control but was not correlated with the severity of retinopathy indicates that other systemic factors may have a greater role in the pathogenesis of this condition. Hence, conventional treatment for macular edema may not be successful in this form of diabetic macular edema.

The exact pathogenesis of diffuse diabetic macular edema has not been elucidated, although a breakdown of the inner blood-retinal barrier is suspected. The possibility of endogenous permeability factors released by the ischemic retina has also been suggested. Endogenous factors include VEGF, prostaglandins, and a host of other factors which are known mediators of vascular permeability. ${ }^{11}$ Corticosteroids have long been used in the treatment of cystoid macular edema because of their ability to inhibit the arachidonic acid pathway, of which prostaglandins are a product. Corticosteroids may also downregulate the production of VEGF. Triamcinolone acetonide, a corticosteroid, has been shown to reduce breakdown of the blood-retinal barrier by the above-mentioned mechanism. Anti-VEGF agents like bevacizumab and ranibizumab are known to cause pan VEGF blockade, while pegabtinib sodium causes selective anti-VEGF blockade and has a role in inhibiting VEGF-mediated vascular endothelial permeability, as shown in experimental and clinical trials. Bevacizumab by virtue of its proven potency, low cost, and longer half-life, seems to be an ideal anti-VEGF agent in the treatment of diffuse macular edema, so was used in this study.

When patients with submacular detachment were analyzed for the presence of other associated systemic comorbidity, $82 \%$ had overt diabetic nephropathy or microalbuminuria; $61 \%$ of these cases were detected for the first time in our study to have significant microalbuminuria/ gross albuminuria. In view of this strong association, it may be concluded that all patients with DSMD should be investigated for microalbuminuria or overt renal disease. Identification and management of this condition may have a bearing on resolution of their macular edema. Also, around one third of eyes with DSMD were associated with anemia, hypertension, and dyslipidemia. It is well known that in diabetic patients, other than glycemic control, control of blood pressure, dyslipidemia, anemia, and nephropathy may affect macular thickness and therefore macular edema. ${ }^{7,8}$ However, the high prevalence of nephropathy in this subgroup with diffuse macular edema, ie, DSMD, was not reported in earlier studies.

It was also observed that submacular fluid absorption was complete in only six of the 14 eyes $(42.9 \%)$ treated with intravitreal bevacizumab and nine of the 20 eyes (45\%) treated with intravitreal triamcinolone. These eyes had the best visual gain and also the best reduction in macular thickness. These data also indicate that systemic control may be as important as ocular therapy in the management of these pathologies. Because this study was performed over a period of 3 months, the above objective may not have been entirely met. Early management of systemic complications like dyslipidemia and nephropathy was not possible in our study population due to lack of awareness, financial issues, and genetic predisposition. Only four of the 11 patients with dyslipidemia were on statins, and the rest were on dietary modification or alternative forms of medicine.

Optical coherence tomographic features seen in the study eyes included retinal edema with cystic spaces (64.7\%) and spongy edema (88.2\%), in addition to subretinal fluid/ DSMD. Other studies have reported retinal edema with cystic spaces in $12 \%-55 \%$ of eyes and subretinal fluid in $10 \%-70 \%$ of eyes with significant diabetic macular edema. ${ }^{2,6}$ Ozdek et $\mathrm{al}^{6}$ showed that $63 \%$ of the subretinal fluid seen on optical coherence tomography was not detected on 
slit-lamp examination and not evident on fundus fluorescein angiography. Kim et $\mathrm{al}^{2}$ also reported that eyes having retinal edema with cystic spaces and subretinal fluid had worse vision than most other subgroups. Ozdemir et $\mathrm{al}^{4}$ reported that $31 \%$ of eyes with retinal edema and cystic spaces had associated subfoveal fluid and poor vision.

Although both intravitreal triamcinolone and bevacizumab improved vision in eyes with DSMD, achieving a reduction in macular thickness at 1 month and demonstrating identical short-term efficacy, the former had a more long-lasting effect on maintaining this visual gain as well as macular thickness at 3 months. Various studies have reported the short-term efficacy of intravitreal bevacizumab in diabetic macular edema. Sohelial et $\mathrm{al}^{12}$ reported that patients treated with intravitreal bevacizumab showed improved vision at all follow-up visits over 36 weeks. A significant reduction in central foveal thickness was observed in only up to 6 weeks, but changes in central foveal thickness did not correlate with gains in vision at all visits. Seo and Park ${ }^{13}$ reported that intravitreal bevacizumab injection resulted in significant improvement in best-corrected visual acuity and central retinal thickness as early as 1 week after injection in patients with diabetic macular edema, and that this beneficial effect persisted for up to 3 months. Haritoglou et $\mathrm{al}^{14}$ found that the increase in visual acuity at 6 weeks after intravitreal bevacizumab could be predicted best by baseline visual acuity. No other factors investigated, including age, central foveal thickness, or previous treatments, were predictive of an increase in visual acuity. Arevalo et $\mathrm{al}^{15}$ reported that the mean number of intravitreal bevacizumab injections required per eye over a 12-month period was three (range 1-6), at a mean interval of $14.1 \pm 10.5$ weeks.

The above studies show that the effect of intravitreal bevacizumab is short-lasting at 6-12 weeks and visual change may not correlate with changes in central foveal thickness. These studies also included eyes that had been treated earlier with laser/other agents, so cannot be compared directly with the results of our study, which only included previously untreated eyes. In eyes with diabetic macular edema which had not been previously treated, Lam et $\mathrm{al}^{16}$ showed that 3-monthly intravitreal bevacizumab injections resulted in a significant reduction in central foveal thickness and improvement in best-corrected visual acuity. There are only a few studies directly comparing the efficacy of intravitreal triamcinolone and bevacizumab in diabetic macular edema. Paccola et $\mathrm{al}^{17}$ demonstrated that central foveal thickness was significantly reduced in an intravitreal triamcinolone group compared with a bevacizumab group at weeks $4,8,12$, and 24 . Visual acuity was significantly higher at weeks 8 and 12 in the intravitreal triamcinolone group compared with the bevacizumab group. Faghihi et al ${ }^{18}$ observed that, at 16 weeks, the change in central foveal thickness observed earlier with intravitreal bevacizumab was not stable, but intravitreal triamcinolone maintained its superior status to laser. At week 16, visual acuities were essentially unchanged for laser and intravitreal bevacizumab, and improvement on intravitreal triamcinolone was marginal. Shukla et a ${ }^{19}$ reported that when serous macular detachment responds to intravitreal triamcinolone acetonide in direct proportion to the height of the serous macular detachment, the response starts to fade by 3 months. Avdin et $\mathrm{al}^{20}$ reported that laser therapy improved visual acuity after intravitreal triamcinolone for significant diabetic macular edema, but not when the treatments were given concomitantly. However, the Diabetic Retinopathy Clinical Research Network study $^{21}$ did not observe a long-term benefit of intravitreal triamcinolone relative to focal/grid photocoagulation in patients with diabetic macular edema.

Many studies have reported a positive correlation of central foveal thickness and visual acuity. The Diabetic Retinopathy Clinical Research network ${ }^{21}$ reported that central foveal thickness correlates modestly with visual acuity in patients with macular edema. However, a wide range of visual acuity values may be observed for a given degree of retinal edema. In this study, $7 \%-17 \%$ of patients showed paradoxical improvement in vision with increased central foveal thickness, and $18 \%-26 \%$ showed a paradoxical worsening of visual acuity with a decrease in central foveal thickness. The same study also did not address whether short-term fluctuations in findings on optical coherence tomography are predictive of long-term effects on visual acuity. Both the United Kingdom Prospective Diabetes Study and Diabetes Control and Complications Trial failed to demonstrate short-term fluctuations in diabetic retinopathy, and beneficial effects were pronounced at longer follow-up. ${ }^{22,23}$ Because ours was a short-term study lasting only 3 months, we believe that systemic factors would not have influenced the results because the study period was too short for this to be the case.

The major limitation of our study is its relatively short duration of follow-up. Although both the study interventions appeared to have a rapid onset of effect, and positive anatomic and visual responses were apparent at 1 month post treatment, the triamcinolone-treated eyes showed a persistent benefit through 3 months of follow-up. Whether this benefit would have persisted longer is unclear. The positive response 
noticed in the study was also influenced by the fact that laser therapy was added to intravitreal pharmacotherapy. However, because both the groups received laser, this influence, even if present, can be ignored. The role of management of systemic disease could have influenced the results but, because of the short study duration, this cannot be confirmed. Although certain injection-related complications would be expected to predominate during the study interval, complications related to corticosteroid medication, such as cataract progression and glaucoma, would be expected to occur over a longer period.

In conclusion, DSMD is a distinct form of diabetic macular edema with a different clinical systemic profile and responds to a modified therapeutic approach rather than conventional photocoagulation. The presence of systemic comorbidity should be investigated in all cases of DSMD. In this study, $82 \%$ of eyes were from patients with overt diabetic nephropathy or microalbuminuria. Considering this high association, it may be concluded that all patients with DSMD should be investigated for microalbuminuria or overt renal disease. Identification and management of this condition may have a bearing on resolution of macular edema. Also, around one third of eyes with DSMD were from patients with anemia, hypertension, and/or dyslipidemia. Combination intravitreal pharmacotherapy and laser treatment seems to be an effective management strategy in these eyes. Intravitreal triamcinolone combined with laser produces a more sustained decrease in macular thickness and improvement in vision than bevacizumab combined with laser. A larger prospective study with a longer follow-up duration is necessary to validate these results.

\section{Disclosure}

The authors report no conflicts of interest in this work.

\section{References}

1. [No authors listed]. Early Treatment Diabetic Retinopathy Study report number 1. Early Treatment Diabetic Retinopathy Study Research Group. Photocoagulation for diabetic macular edema. Arch Ophthalmol. 1985;103:1796-1806.

2. Kim BY, Smith SD, Kaiser PK. Optical coherence tomographic patterns of diabetic macular edema. Am J Ophthalmol. 2006;142:405-412.

3. Panozzo G, Parolini B, Gusson E, et al. Diabetic macular edema - an OCT based classification. Semin Ophthalmol. 2004;19:13-20.

4. Ozdemir H, Karacorlu M, Karacorlu S. Serous macular detachment in diabetic cystoid macular edema. Acta Ophthalmol Scand. 2005;83: 63-66.

5. Thomas D, Bunce C, Moorman C, et al. Frequency and associations of a taut thickened hyaloid, partial vitromacular separation and subretinal fluid in patients with diabetic macular edema. Retina. 2005;25:883-888.
6. Ozdek SC, Erdinc A, Gurelik G. Optical coherence tomographic assessment of diabetic macular edema: comparison with fluorescein angiographic and clinical findings. Ophthalmologica. 2005;219:86-92.

7. Klein R, Klein BEK, Moss SE, et al. The Wisconsin Epidemiologic Study of Diabetic Retinopathy: XV. The long-term incidence of macular edema. Ophthalmology.1995;102:7-16.

8. Davis MD, Fisher MR, Gangnon RE, et al. Risk factors for high-risk proliferative diabetic retinopathy and severe visual loss: Early Treatment Diabetic Retinopathy Study. Report 18. Invest Ophthalmol Vis Sci. 1998:39:233-252.

9. Alkuraya H, Kangave D, Abu El-Asrar AM. The correlation between optical coherence tomographic features and severity of retinopathy, macular thickness and visual acuity in diabetic macular edema. Int Ophthalmol. 2005;26:93-99.

10. Fang X, Sakaguchi H, Gomi F, et al. Efficacy and safety of one intravitreal injection of bevacizumab in diabetic macular oedema. Acta Ophthalmol. 2008;86:800-805.

11. Funatsu H, Yamashita H, Noma H, et al. Increased levels of vascular endothelial growth factor and interleukin-6 in the aqueous humor of diabetics with macular edema. Am J Ophthalmol. 2002;133:70-77.

12. Soheilian M, Ramezani A, Obudi A, et al. Randomized trial of intravitreal bevacizumab alone or combined with triamcinolone versus macular photocoagulation in diabetic macular edema. Ophthalmology. 2009;116:1142-1150

13. Seo JW, Park IW. Intravitreal bevacizumab for treatment of diabetic macular edema. Korean J Ophthalmol. 2009;23:17-22.

14. Haritoglou C, Kook D, Neubauer A, et al. Intravitreal bevacizumab (Avastin) therapy for persistent diffuse diabetic macular edema. Retina . 2006;26:999-1005.

15. Arevalo JF, Sanchez JG, Wu L, et al. Primary intravitreal bevacizumab for diffuse diabetic macular edema: the Pan-American Collaborative Retina Study Group at 24 months. Ophthalmology. 2009;116: 1488-1497.

16. Lam DS, Lai TY, Lee VY, et al. Efficacy of 1.25 MG versus $2.5 \mathrm{MG}$ intravitreal bevacizumab for diabetic macular edema: six-month results of a randomized controlled trial. Retina. 2009;29:292-299.

17. Paccola L, Costa RA, Folgosa MS, et al. Intravitreal triamcinolone versus bevacizumab for treatment of refractory diabetic macular oedema (IBEME study). Br J Ophthalmol. 2008;92:76-80.

18. Faghihi H, Roohipoor R, Mohammadi SF, et al. Intravitreal bevacizumab versus combined bevacizumab-triamcinolone versus macular laser photocoagulation in diabetic macular edema. Eur JOphthalmol. 2008;18: 941-948.

19. Shukla D, Behera UC, Chakraborty S, et al. Serous macular detachment as a predictor of resolution of macular edema with intravitreal triamcinolone injection. Ophthalmic Surg Lasers Imaging. 2009;40: 115-119.

20. Aydin E, Demir HD, Yardim H, et al. Efficacy of intravitreal triamcinolone after or concomitant with laser photocoagulation in nonproliferative diabetic retinopathy with macular edema. Eur J Ophthalmol. 2009;19: 630-637.

21. Beck RW, Edwards AR, Aiello LP, et al; Diabetic Retinopathy Clinical Research Network (DRCR.net). Three-year follow-up of a randomized trial comparing focal/grid photocoagulation and intravitreal triamcinolone for diabetic macular edema. Arch Ophthalmol. 2009;127: 245-251.

22. Diabetic Control and Complications Trial Research Group. Progression of retinopathy with intensive versus conventional treatment in the Diabetic Control and Complications Trial. Ophthalmology. 1995;102: 647-661.

23. UK Prospective Diabetes Study Group. UKPDS 33 Intensive blood glucose control with sulphonylureas or insulin compared with conventional treatment and risk of complications in patients with type 2 diabetes. Lancet. 1998;352:837-853. 


\section{Publish your work in this journal}

Clinical Ophthalmology is an international, peer-reviewed journal covering all subspecialties within ophthalmology. Key topics include: Optometry; Visual science; Pharmacology and drug therapy in eye diseases; Basic Sciences; Primary and Secondary eye care; Patien Safety and Quality of Care Improvements. This journal is indexed on Submit your manuscript here: http://www.dovepress.com/clinical-ophthalmology-journal
Dovepress

PubMed Central and CAS, and is the official journal of The Society of Clinical Ophthalmology (SCO). The manuscript management system is completely online and includes a very quick and fair peer-review system, which is all easy to use. Visit http://www.dovepress.com/ testimonials.php to read real quotes from published authors. 